\title{
The association between cognitive function and objective adherence to dietary sodium guidelines in patients with heart failure
}

This article was published in the following Dove Press journal:

Patient Preference and Adherence

2 March 2016

Number of times this article has been viewed

\author{
Mary A Dolansky' \\ Julie T Schaefer ${ }^{2}$ \\ Misty AW Hawkins ${ }^{3}$ \\ John Gunstad ${ }^{2}$ \\ Anup Basuray ${ }^{4}$ \\ Joseph D Redle ${ }^{5}$ \\ James C Fang ${ }^{6}$ \\ Richard A Josephson 7,8 \\ Shirley M Moore' \\ Joel W Hughes ${ }^{2}$ \\ 'School of Nursing, Case Western \\ Reserve University, Cleveland, $\mathrm{OH}$ \\ USA; '2Department of Psychology, \\ Kent State University, Kent, OH, USA; \\ ${ }^{3}$ Department of Psychology, Oklahoma \\ State University, Stillwater, OK, USA; \\ ${ }^{4}$ Ohio Health Heart and Vascular \\ Physicians, Columbus, OH, USA; \\ ${ }^{5}$ Department of Cardiology, Summa \\ Health System, Akron City Hospital, \\ OH, USA; ${ }^{6}$ Department of Internal \\ Medicine, Division of Cardiology, \\ University Hospital, Salt Lake City, \\ UT, USA; ${ }^{7}$ Department of Medicine, \\ Case Western Reserve University, \\ Cleveland, OH, USA; ${ }^{8}$ Harrington \\ Heart \& Vascular Institute, University \\ Hospitals, Cleveland, OH, USA
}

Correspondence: Mary A Dolansky School of Nursing, Case Western Reserve University, 2120 Cornell Road Cleveland, OH 44I06, USA

Tel +l 2163680568

EmailmadI5@case.edu
Background: Although cognitive impairment is common in heart failure (HF) patients, its effects on sodium adherence recommendations are unknown.

Purpose: Our aim is to examine if cognitive function is associated with patient sodium adherence. Methods: Sodium collection/excretion and cognitive function were assessed for $339 \mathrm{HF}$ patients over a 5-8-week period. Neuropsychological testing was performed at baseline (Visit 1), whereas two 24-hour urine samples were collected within 7 weeks postbaseline. The ability to collect two 24-hour urine samples and the estimation of sodium excretion levels from these samples were used to estimate sodium adherence recommendations.

Results: Nearly half (47\%) of the study participants $(n=159)$ were unable to give two valid 24-hour urine samples. Participants who were unable to adhere to two valid 24-hour urine samples had significantly poorer attention and global cognition tests $(P<0.044)$, with a trend for poorer executive function $(P=0.064)$. Among those with valid samples, urine sodium level was not associated with global cognitive function, attention, executive function, or memory after adjusting for covariates. Female sex was associated with lower sodium excretion (all $P<0.01$ ); individuals with knowledge of sodium guidelines had less intake of sodium, resulting in excretion of less sodium (all $P \leq 0.03$ ). Conversely, higher socioeconomic status (SES) and body mass index (BMI) were associated with greater sodium (all $P \leq 0.02$ and $P \leq 0.01$ ).

Conclusion: Adherence to urine sodium collection was poor, especially among those with poorer cognitive function. Sodium consumption exceeded recommended amounts and was unrelated to cognitive function. Interventions for improving sodium adherence should focus on at-risk groups (high SES and BMI) and at improving knowledge of recommended salt intake. Keywords: urine collection, salt intake, attention, executive function, memory, cardiac

\section{Introduction}

Heart failure (HF) impacts nearly 6 million individuals in the United States $^{1}$ and is associated with decreased quality of life, ${ }^{2}$ reduced physical function, ${ }^{3}$ and cognitive impairment across multiple domains, including attention, executive function, and memory. ${ }^{4}$ Patients with $\mathrm{HF}$ are also at increased risk for more severe neurodegenerative conditions, such as Alzheimer's disease and other dementias. ${ }^{5}$ These HF-related cognitive deficits may be problematic as the treatment regimen for HF is complex, requiring multiple medications, daily weighing, and adherence to a low-sodium diet. For example, cognitive impairment has been shown to predict poorer adherence to a variety of HF-treatment-related behaviors (eg, attending provider appointments and medication management), ${ }^{6-8}$ and poorer adherence can contribute to poorer HF outcomes (eg, greater adverse coronary events, hospitalizations, and all-cause mortality). ${ }^{9-11}$ Unfortunately, many of these 
studies examine self-reported adherence behaviors. Given the probability of underestimation, ${ }^{12}$ self-report can be particularly problematic for assessing adherence to HF dietary guidelines, such as sodium restriction.

The average daily American diet contains more than 4,000 mg of sodium. ${ }^{13,14}$ This amount may lead to an exacerbation of symptoms as well as other adverse effects in patients with HF. ${ }^{15}$ The adverse effects center around the altered sodium homeostasis observed in the HF state ${ }^{16}$ - sodium and water retention despite fluid overload - and occur primarily because of the negative physiologic impact of sodium on many interrelated cardiovascular functions, including blood pressure, ${ }^{17}$ arterial wall stiffness, ${ }^{18}$ and G protein-coupled receptor kinase 2 signaling. ${ }^{19}$ Thus, the Heart Failure Society of America suggests 2,000-3,000 mg or less of daily sodium in the HF population, depending on HF severity. ${ }^{15}$ The American Heart Association (AHA) initially recommended an even more stringent $1,500 \mathrm{mg}$ or less for all individuals regardless of a history of cardiac disease. ${ }^{20}$ The variability of these guidelines is, in part, due to a lack of robust data. As a result, the most recently updated the American College of Cardiology Foundation (ACCF) and AHA Heart Failure Guidelines have downgraded sodium restriction in $\mathrm{HF}$ patients to a maximum of 2,000 $\mathrm{mg} / \mathrm{d}$ and have limited the $1,500 \mathrm{mg}$ restriction to Stage A and B HF only. ${ }^{21}$

Previous work from the Heart Failure Adherence, Behavior, and Cognition Study (Heart ABC) has demonstrated that adherence to the sodium restriction guidelines is poor among patients with $\mathrm{HF}^{22}$ Specifically, when using a less stringent sodium restriction guideline (ie, $<3,000 \mathrm{mg}$ sodium intake) over half of the study cohort ( $52 \%$ ) failed to meet the guideline. Only $23 \%$ met the more stringent $2,000 \mathrm{mg} / \mathrm{d}$ maximum, indicating that only one of every four patients with HF are meeting the recommended sodium intake. ${ }^{22}$ Although the sodium restriction values may vary slightly across studies, the high nonadherence rates are consistent, ranging from $52 \%$ in our study to $66 \%$ in others. ${ }^{23,24}$ Individuals with cognitive impairment may have even greater difficulties with meeting the sodium recommendations, given that adherence to the guidelines requires multiple cognitive resources (eg, memory and executive function) in order to remember the recommended sodium levels, attend to and process information from food labels and products to ascertain sodium content, persist at a low-sodium diet, and inhibit consumption of high-sodium products. However, this assumption has not been directly tested in previous studies. Thus, the objective of the current study was to extend previous work in the Heart ABC sample by examining whether poorer cognitive function is associated with poorer sodium adherence among this community-based sample of adults with HF. Two major strengths of the current study are the use of objective and gold standard measures for cognitive function and sodium adherence, including a comprehensive, validated neuropsychological battery and two 24-hour urine collections with measured sodium excretion. Using a full neuropsychological battery provides more detailed information than commonly used cognitive screens, ${ }^{25,26}$ and objective sodium excretion is less prone to the inaccuracy and underestimation, which is common for self-reported sodium intake. ${ }^{12}$ We hypothesized that greater cognitive deficits would be associated with poorer adherence to dietary sodium guidelines, as measured by reduced ability to collect urine sodium and greater 24-hour urine sodium excretion.

\section{Methods}

\section{Study population}

The total sample consisted of 372 outpatients with HF enrolled in the Heart ABC study. Detailed information about eligibility requirements and inclusion/exclusion criteria have been published previously. ${ }^{22,27}$ In order to be selected for the current analyses, participants had to have complete data on the cognitive variables and urinary sodium $(\mathrm{N}=339)$. Participants were predominantly older, male, white, and had at least a high-school education (Table 1). The majority of the sample was categorized as having NYHA (New York Heart Association) Class III HF severity. The clinical trial registration number for this study is $\underline{\mathrm{NCT} 01461629 .}$.

\section{Measures}

\section{Cognitive functioning}

Cognitive functioning was measured across multiple domains using neuropsychological tests that have strong validity and reliability. The four cognitive domains were as follows:

1) Global cognitive function: General cognitive ability across a variety of domains. Global cognitive function was examined with the Modified Mini-Mental Status Examination (3MS). ${ }^{28}$ Scores range from 0 to 100 with higher scores indicative of better cognitive performance. Scores $\leq 90$ on the $3 \mathrm{MS}$ are indicative of some degree of cognitive impairment. ${ }^{29}$

2) Attention: The capacity to attend to and process information. Attention was measured by the Stoop Word and Color subtests, ${ }^{30}$ Trail Making Test A, ${ }^{31}$ and LetterNumber Sequencing. ${ }^{32}$

3) Executive function: The capacity to problem-solve, plan, inhibit, and reason. Executive function was assessed using 
Table I Descriptive statistics of full sample $(\mathrm{N}=339)$

\begin{tabular}{|c|c|c|c|}
\hline Study variable & $\begin{array}{l}\text { Participants without two adequate } \\
\text { urine samples }(n=\mid 59)\end{array}$ & $\begin{array}{l}\text { Participants with two adequate } \\
\text { urine samples }(n=180)\end{array}$ & $P$-value \\
\hline Average sodium intake $(\mathrm{mg})$, mean $(\mathrm{SD})$ & $\square^{\mathrm{a}}$ & $3,152.34(1,175.35)$ & $-^{\mathrm{a}}$ \\
\hline \multicolumn{4}{|l|}{ NYHA, n (\%) } \\
\hline Class I & $18(1 \mid .32)$ & $17(9.44)$ & \\
\hline Class II & $35(22.01)$ & $47(26.11)$ & \\
\hline Class III & $97(6 I .0 I)$ & $107(59.44)$ & \\
\hline Class IV & $9(5.66)$ & $9(5.00)$ & 0.807 \\
\hline Qualifying ejection fraction & 30.00 (8.29) & $29.09(8.50)$ & 0.320 \\
\hline Age, mean (SD) & $69.14(10.29)$ & $68.72(8.92)$ & 0.690 \\
\hline \multicolumn{4}{|l|}{ Sex, n (\%) } \\
\hline Male & $96(60.38)$ & III (6I.67) & \\
\hline Female & $63(39.62)$ & $69(38.33)$ & 0.808 \\
\hline \multicolumn{4}{|l|}{ Minority status, n (\%) } \\
\hline White & III (69.8I) & I 34 (74.44) & \\
\hline Non-white & $48(30.19)$ & $46(25.56)$ & 0.342 \\
\hline \multicolumn{4}{|l|}{ Education, n (\%) } \\
\hline 8th grade or less & $5(3.14)$ & $4(2.22)$ & \\
\hline 9th-I Ith grade & $19(11.95)$ & $12(6.67)$ & \\
\hline High school & $49(30.82)$ & $45(25.00)$ & \\
\hline Technical or trade school & $15(9.43)$ & $18(10.00)$ & \\
\hline Some college & $40(25.16)$ & $55(30.56)$ & \\
\hline Bachelor's degree & $17(10.69)$ & $29(16.11)$ & \\
\hline Master's degree & $14(8.8 \mathrm{I})$ & $17(9.44)$ & 0.355 \\
\hline SES, mean (SD) & $-0.14(4.74)$ & $0.22(4.07)$ & 0.461 \\
\hline \multicolumn{4}{|l|}{ Impaired health literacy, n (\%) } \\
\hline No & 75 (47.47) & $99(55.62)$ & \\
\hline Yes & $83(52.53)$ & $79(44.38)$ & 0.136 \\
\hline \multicolumn{4}{|l|}{ Someone else prepares food, n (\%) } \\
\hline No & $93(58.49)$ & $94(52.22)$ & \\
\hline Yes & $66(4 I .5 I)$ & $86(47.78)$ & 0.247 \\
\hline \multicolumn{4}{|l|}{ Someone else brings food, n (\%) } \\
\hline No & $39(24.53)$ & $36(20.00)$ & \\
\hline Yes & $120(75.47)$ & $144(80.00)$ & 0.316 \\
\hline \multicolumn{4}{|l|}{ Can correctly recall the sodium } \\
\hline \multicolumn{4}{|l|}{ Recommendation, $\mathrm{n}(\%)$} \\
\hline No & $130(81.76)$ & $131(73.60)$ & \\
\hline Yes & $29(18.24)$ & $47(26.40)$ & 0.073 \\
\hline BMI, mean (SD) & $29.93(7.02)$ & $30.10(6.40)$ & 0.815 \\
\hline Comorbidity, mean (SD) & $3.37(\mathrm{I} .6 \mathrm{I})$ & $3.33(1.88)$ & 0.821 \\
\hline No of past hospitalizations for HF, mean (SD) & $2.19(2.05)$ & $2.02(1.96)$ & 0.442 \\
\hline Depression, mean (SD) & $4.69(5.17)$ & $4.26(4.44)$ & 0.417 \\
\hline Anxiety, mean (SD) & $13.19(5.70)$ & $12.21(4.58)$ & 0.083 \\
\hline Social support, mean (SD) & $68.61(13.87)$ & $69.64(14.06)$ & 0.501 \\
\hline \multicolumn{4}{|l|}{ Cognitive domains, mean (SD) } \\
\hline Attention & $43.54(7.47)$ & $45.22(7.44)$ & $0.044^{*}$ \\
\hline Memory & $47.21(8.25)$ & $48.11(7.53)$ & 0.298 \\
\hline Executive function & $45.06(8.23)$ & $46.75(7.87)$ & 0.064 \\
\hline Global cognition & $90.92(7.04)$ & $92.71(6.15)$ & $0.014^{*}$ \\
\hline
\end{tabular}

Notes: a Value not indicated due to invalid urine collection. $* P<0.05$.

Abbreviations: BMI, body mass index; HF, heart failure; NYHA, New York Heart Association; SES, socioeconomic status; SD, standard deviation.

the Stroop Color Word subtest, ${ }^{30}$ Trail Making Test B, ${ }^{31}$ and the Frontal Assessment Battery. ${ }^{33}$

4) Memory: The capacity to retain and recall verbal information. Memory was measured using the Rey Auditory Verbal Learning Test Learning Over Time, True Hits, Short Delay, and Long Delay scores. ${ }^{34}$
Raw neuropsychological test scores for the individual attention, executive function, and memory tests were converted to age-adjusted scaled scores using normative data for each test. To facilitate interpretation, these scaled scores were converted to $T$-scores $(M=50$, standard deviation $[\mathrm{SD}]=10)$. The relevant $T$-scores were then averaged to create a 
composite $T$-score for each domain: attention, executive function, and memory. $T$-scores $\leq 35$ indicate impaired cognitive functioning. ${ }^{35}$ Global cognitive function was measured by a single test (the $3 \mathrm{MS}$ ), so no composite was created.

Sodium adherence was conceptualized as follows: 1) the ability to collect two adequate 24-hour urine samples and 2 ) the average amount of sodium ( $\mathrm{mg}$ ) excreted in two samples. Both of these variables were considered because the validity of the sodium excretion adherence variable depends directly on the patients' ability to adhere to the urine collection methods. The collection methods included giving patients a collection device, urine jug, and verbal/written instructions to collect all urine within a 24-hour window at two separate visits. Participants were instructed to store each sample in the refrigerator and document collection start and stop times. A telephone reminder call was also given 1 day prior to each collection to ensure protocol fidelity. In addition, urine samples were analyzed for creatinine output in the same 24-hour window in order to ensure the adequacy of the samples. Creatinine values in each sample were compared to previously published ranges of creatinine excretion that would be expected based on sex, race, and weight. ${ }^{36}$ Those with creatinine values in the appropriate range were marked as adequate samples. Thus, the "ability to collect two adequate samples" was defined dichotomously ( $1=$ Yes, $0=$ No). Participants were coded as "Yes" if they provided the research assistant with two urine jugs containing 24-hour urine specimens and expected normative 24-hour creatinine excretion levels based on their age, sex, race, and weight. ${ }^{22}$ The average sodium excretion from the two adequate samples was used to estimate 24-hour sodium intake. Two 24-hour collections were used to account for day-to-day variability in intake. Although an indirect measure, urinary sodium excretion is considered a gold standard estimate of dietary sodium intake and has been shown to be highly correlated with dietary intake in non-HF patients, with $>95 \%$ of intake being excreted in the urine in temperate climates. ${ }^{37}$ Urinary sodium excretion may provide a more accurate assessment of dietary sodium, especially as compared to food diaries, which often result in an underestimation of sodium consumption. ${ }^{12,38}$

\section{Covariates}

The following variables were included as covariates/ potential confounders of any observed relationship between cognitive function and sodium excretion: age (years), sex $(0=$ male, $1=$ female $)$, minority status $(0=$ white, $1=$ minority status), education level ( $1=8$ th grade or less, $2=9-11$ th grade, $3=$ high school, $4=$ technical or trade school, 5= some college,
$6=$ bachelor's degree, $7=$ master's degree), socioeconomic status (SES), Charlson Comorbidity Index (CCI) score, ${ }^{39}$ selfreported HF severity level (as estimated using self-reported symptoms and applying the NYHA criteria), ${ }^{40}$ overall health literacy, depression, anxiety, social support, food preparation/delivery status, and knowledge of the sodium intake recommendation. SES was estimated using subjects' zip code. ${ }^{41}$ The SES score was calculated as a $z$-score using indicators of income and education for each zip code, with higher scores indicating higher SES. The CCI is a summary score of comorbid medical conditions (eg, diabetes, peripheral vascular disease, myocardial infarction, etc). ${ }^{39}$ NYHA levels of HF severity are classified as Class I (Mild), Class II (Mild), Class III (Moderate), and Class IV (Severe) and based on the degree of exertion needed to elicit HF symptoms; ${ }^{40}$ in this study, we used patients' self-reported HF symptoms for classification. Overall health literacy was measured with the Medical Term Recognition Test (METER). ${ }^{42}$ Depressive symptoms were assessed using the Patient Health Questionnaire-9 (PHQ-9). ${ }^{43}$ Anxiety was estimated with the 7-item Patient Reported Outcomes Measurement Information System Anxiety Subscale (PROMIS-Anxiety). ${ }^{44}$ Social support was quantified using the Multidimensional Scale of Perceived Social Support (MSPSS). ${ }^{45}$ Two dichotomous questions were asked to determine whether anyone in the household helped the patient with food preparation (Yes/No) or delivered food to the patients (Yes/No). To assess whether the participant knew the sodium intake recommendation at study entry, we asked participants: "What is the maximum amount of sodium you should consume in a day?" The correct response was "no more than $2,000 \mathrm{mg}$ or one teaspoon of salt" and was coded as a score of 1 , whereas incorrect responses were coded as 0 . Those who answered incorrectly were given brief education about the sodium guidelines.

\section{Procedure}

All patients were recruited from inpatient and/or outpatient cardiology practices in northeast Ohio and gave their written, informed consent to participate. All procedures were approved by the Institutional Review Boards of Kent State University, Summa Health Systems, Inc., and University Hospitals Case Medical Center. After recruitment and written consent, a trained research assistant conducted four visits to the patients' homes over a 5-8-week period, with 6 weeks as standard from Visit 1 (baseline) to Visit 4. First, neuropsychological testing and self-report questionnaires were administered, and education on adhering to a 2,000 mg sodium diet was provided at the first home visit (baseline). At the 
second home visit (1-2 weeks after baseline) and the third home visit (1-2 weeks after Visit 2), participants were given the urine collection implements with instructions to collect all urine within a 24-hour window. As a reminder, subjects were telephoned 1 day prior to collection. Participants were instructed to keep samples cold by storage in refrigerators and were responsible for reporting the start and stop times of collection. Study personnel retrieved the urine samples during the third and fourth home (3-4 weeks after Visit 3) visits. Samples were transported under ambient temperatures to the clinical research lab.

\section{Analyses}

To assess if greater cognitive deficits or other demographic and biopsychosocial variables were associated with reduced ability to collect urine sodium (Yes/No), chi-square analysis and $t$-tests were conducted. Next, simple linear regressions were performed to determine which variables predicted average sodium excretion. Significant biopsychosocial predictors from the simple linear regressions were then entered together as covariates into a set of four simultaneous regression models to determine which variables uniquely predicted sodium excretion. Each of the four cognitive domains was used as a predictor variable in a separate regression to avoid multicollinearity in each model.

\section{Results \\ Participants}

The sample consisted of predominantly older, Caucasian males with NYHA Class III HF and reduced ejection fraction. Although the cognitive performance of the sample was average to low-average (Table 1), between 6\% and 12\% exhibited clinically meaningful cognitive impairment, as assessed using a cutoff of $\leq 35$ for the $T$-score composition: $6.0 \%$ memory impaired, $9.7 \%$ executive function impaired, and $11.9 \%$ attention impaired. The majority of the sample (85\%) failed to meet the ACCF/AHA Heart Failure Guidelines of less than 2,000 $\mathrm{mg}$ of sodium per day. On average, the 24-hour sodium intake was 3,152.34 ( $\mathrm{SD}=1,175.35$; range: $897.00-7,233.50) \mathrm{mg}$ (Table 1 ). Further, only $26.4 \%$ $(n=47)$ of the sample had existing knowledge of the sodium intake recommendation (Table 1).

\section{The ability to collect a valid urine sample}

Nearly half $(47 \%)$ of the study participants $(n=159)$ were unable to give two valid 24-hour urine samples. Participants who were unable to collect two valid 24-hour urine samples had significantly lower scores on the attention and global cognition tests $(P<0.044)$ (Table 1$)$. A trend was observed for lower executive function scores and poorer adherence to urine collection $(P=0.064)$, whereas memory was not associated with urine collection. All further analyses of sodium excretion were conducted using data only from those participants with two adequate 24-hour urine samples to ensure the most accurate estimation of 24-hour sodium intake.

\section{Average amount of sodium excretion}

To assess whether poorer cognitive function was associated with greater sodium excretion, simple linear regression was first conducted; then, all significant predictors and one variable that was trending toward significance (race) were entered into an adjusted model. All data analyses were conducted using SAS version 9.2 statistical software (SAS Institute, Cary, NC, USA).

Simple linear regression results revealed that none of the cognitive domains were associated with sodium intake (Table 2). Specifically, attention, executive function, memory, and global cognitive function failed to predict 24-hour urinary sodium excretion (all $P \geq 0.48$ ). Female sex $(P<0.01)$, having someone else prepare food $(P=0.03)$, and

Table 2 Simple linear regression of factors predicting average sodium excretion $(\mathrm{mg})(\mathrm{N}=180)$

\begin{tabular}{|c|c|c|}
\hline Factors & $b(S D)$ & $P$-value \\
\hline NYHA & $-92.12(120.60)$ & 0.446 \\
\hline Age & $-10.18(9.85)$ & 0.303 \\
\hline Female & $-817.04(170.00)$ & $<0.00 I^{*}$ \\
\hline Non-white & $-343.67(199.76)$ & 0.087 \\
\hline Education & $46.65(57.77)$ & 0.421 \\
\hline SES & $60.62(21.44)$ & $0.005^{*}$ \\
\hline Impaired health literacy & $-95.92(178.32)$ & 0.591 \\
\hline Someone else prepares food & $-370.27(173.67)$ & $0.034 *$ \\
\hline Someone else brings food & $58.38(219.58)$ & 0.791 \\
\hline \multicolumn{3}{|l|}{ Can correctly recall the sodium } \\
\hline Recommendation & $-400.81(199.15)$ & $0.046 *$ \\
\hline BMI & $35.49(13.57)$ & $0.010 *$ \\
\hline Comorbidity & $41.36(46.72)$ & 0.377 \\
\hline No of past hospitalizations for HF & $-17.36(44.27)$ & 0.695 \\
\hline Depression & $4.70(19.84)$ & 0.813 \\
\hline Anxiety & $9.94(19.34)$ & 0.608 \\
\hline Social support & $-6.73(6.24)$ & 0.282 \\
\hline \multicolumn{3}{|l|}{ Cognitive domains } \\
\hline Attention & $-1.92(12.13)$ & 0.875 \\
\hline Memory & $-4.53(11.72)$ & 0.700 \\
\hline Executive function & $8.29(11.62)$ & 0.477 \\
\hline Global cognition & $-7.83(14.47)$ & 0.589 \\
\hline
\end{tabular}

Notes: Each factor above was entered into a separate regression model predicting average sodium excretion. $* P<0.05$.

Abbreviations: BMI, body mass index; HF, heart failure; NYHA, New York Heart Association; SES, socioeconomic status; SD, standard deviation; $b$, unstandardized regression coefficient. 


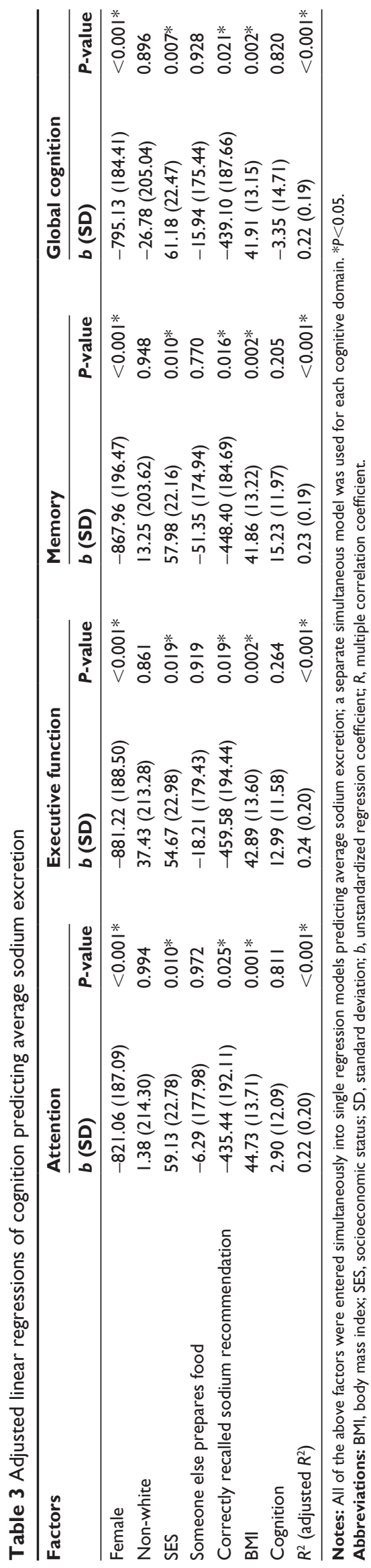

having existing knowledge of the sodium recommendation $(P=0.05)$ predicted lower sodium intake, whereas higher SES $(P=0.01)$ and body mass index (BMI) $(P=0.01)$ predicted greater sodium intake. Female sex and BMI may have predicted sodium due to dietary differences across sex and weight classes. There was a trend toward significance for non-white race predicting lower sodium intake $(P=0.09)$.

All significant predictors and race, which was trending toward significance, were entered into four adjusted models, one for each cognitive domain (Table 3). None of the cognitive domains significantly predicted sodium intake in the adjusted models. In all four models, female sex and having existing knowledge of the sodium recommendation were significantly correlated with lower sodium intake, whereas higher SES and higher BMI were associated with greater sodium intake.

\section{Discussion}

The objective of the current study was to examine whether cognitive impairment was associated with poorer sodium adherence in a community-based sample of older adults with HF. At the group level, participants' cognitive performance was intact, but individual scores varied - with rates of clinical impairment at $6 \%$ for memory, $10 \%$ for executive function, and $12 \%$ for attention. This pattern (ie, more deficits for attention/executive function than for memory) and these rates are comparable to those reported for community-dwelling HF populations $(15 \%-25 \%)^{4,46-48}$ and suggest that our sample exhibited similar range of cognitive performance as previous investigations. Our hypothesis that greater cognitive deficits would be associated with poorer adherence to dietary sodium guidelines was partially supported. Specifically, greater cognitive deficits were associated with failure to collect urine sodium; however, cognition was not related to 24-hour urine sodium excretion. Adherence to urine sodium collection was poor with only one of every two patients (53\%) collecting two adequate urine samples. Those who failed to collect valid samples exhibited poorer global cognition and attention, with a trend toward poorer executive function.

Previous evidence linking cognitive function to treatment adherence in patients with HF is mixed. In some studies, impaired cognitive function in HF patients predicts poorer overall adherence ${ }^{8}$ and self-care behaviors, ${ }^{49}$ but other studies did not support these findings. ${ }^{50}$ Evidence regarding adherence to dietary and sodium recommendations, in particular, is limited. Alosco et $\mathrm{al}^{8}$ found that only impaired language functioning was associated with poorer adherence to self-reported dietary recommendations. Cameron et $\mathrm{al}^{49}$ found that mild 
cognitive impairment was associated with impaired management of HF (ie, ability to recognize, evaluate, and respond to changes in symptoms), but not self-care maintenance behaviors, which include adherence to a low-sodium diet. Contrary to these findings, Dickson et $\mathrm{al}^{51}$ found that cognitive function significantly predicted self-care maintenance behaviors.

Several explanations exist which may explain the discrepancies between studies examining cognitive function and HF treatment adherence. First, the type of treatment adherence being assessed varies widely across studies (eg, medication vs diet vs physician appointment attendance, etc) and may yield differential findings. For example, our study is among the first to investigate the effect of cognitive function on sodium intake adherence in HF patients using the most objective method available to measure dietary sodium intake (ie, sodium excretion). ${ }^{52}$ In contrast, previous studies that examined the effect of cognitive function on self-care have only looked at adherence to general HF guidelines, including daily weighing and medications ${ }^{49}$ or examined these effects using only self-report measures of dietary intake. ${ }^{8}$ A potential explanation for why cognition was associated with the ability to collect urine sample - but not with sodium excretion - may be restricted range. A full $47 \%$ of participants failed to collect an adequate sample, whereas only $15 \%$ of patients with a valid urine sample excreted the recommended $<2,000 \mathrm{mg}$ of sodium. Thus, adherence behaviors, such as urinating into the collection containers for a full 24 hours, show greater variability across participants than dietary restriction of sodium. Such rates suggest that even patients with the cognitive capabilities to obtain an adequate urine specimen struggled with adherence to sodium restriction, regardless of cognitive status and likely due to the sodium-rich foods that comprise the American diet. ${ }^{14}$

Importantly, participants who were able to verbalize at baseline that their doctor instructed them to consume less than 2,000 mg of sodium per day did exhibit significantly lower sodium intake, excreting approximately $400 \mathrm{mg}$ less sodium on average. Others have found that HF knowledge is associated with greater adherence to recommended guidelines for management of symptoms in HF patients ${ }^{53}$ and in those with mild cognitive impairment. ${ }^{54}$ It is possible that those participants who recalled the sodium guidelines had a plan implemented for the reduction of daily sodium. Perhaps more concerning, only $26.40 \%$ of the study sample $(n=47)$ knew the sodium recommendation at baseline. More research is warranted to investigate the impact of recall of recommendations on sodium intake and having a daily plan for self-management in HF patients.
A low-sodium diet is a recommendation endorsed by all national and international $\mathrm{HF}$ guidelines. ${ }^{16}$ Sodium retention has been identified as a leading cause of exacerbation of HF symptoms. ${ }^{55}$ Despite this evidence, few HF patients comply with dietary sodium recommendations as evidenced by other research teams ${ }^{56}$ as well as our sample. Indeed, only $15 \%$ of our sample met the sodium intake recommendations. Understanding which factors contribute to compliance with sodium recommendations in HF patients is crucial to improve clinical HF outcomes. Though cognitive deficits appear to be a logical predictor of adherence to sodium restrictions and have been associated with poorer HF outcomes, this assumption was not supported in the current study.

Although our study has several strengths, including objective measurement of sodium intake/adherence, comprehensive neurocognitive assessment, and a large sample size, limitations should be noted. First, the study was crosssectional, lacked a non-HF control group, and comprised sample that was mostly white males with HF who reside in the USA. Future prospective research is needed that examines whether our results generalize to non-HF populations and to more diverse HF populations. Another limitation is that the urine samples were based on a 24-hour collection period. It is possible that this 24-hour period was not a typical day of dietary sodium intake. Thus, similar studies examining a more diverse sample and a longer urinary sodium collection interval should be conducted to confirm our findings. Next, the lack of objective data regarding HF severity (eg, stenosis or other structural damage) is a limitation. Future studies should collect and utilize this information to determine to which degree physiologic damage to the heart is related to both cognition and adherence metrics. Finally, given that HF patients with others who prepared meals exhibited lower sodium excretion, future studies are needed to clarify the possible interaction between cognitive function and instrumental support of others. It is likely that cognitively impaired persons receive greater assistance from others and thus may be able to better adhere due to this other support.

\section{Conclusion}

In brief conclusion, the majority of patients with HF experience compromised cognitive function. Nearly half of the patients failed to collect adequate urine sample, with greater collection failure among those with poorer cognitive function. Among those with valid urine collection, cognitive impairment was not associated with dietary sodium intake. Significant predictors of greater sodium intake among this group included high SES and high BMI, which may be more 
clinically useful in identifying patients at risk for nonadherence to dietary guidelines in HF.

\section{Acknowledgments}

This work was supported by the National Heart, Lung, and Blood Institute (R01 HL096710-01A1 to MD and JWH).

\section{Disclosure}

The authors report no conflicts of interest in this work.

\section{References}

1. Mozaffarian D, Benjamin EJ, Go AS, et al. Heart disease and stroke statistics - 2015 update: a report from the American Heart Association. Circulation. 2015;131(4):e29-e322.

2. Bennett SJ, Oldridge NB, Eckert GJ, et al. Comparison of quality of life measures in heart failure. Nurs Res. 2003;52(4):207-216.

3. Seo Y, Roberts BL, Piña I, Dolansky M. Predictors of motor tasks essential for daily activities among persons with heart failure. J Card Fail. 2008;14(4):296-302.

4. Pressler SJ, Subramanian U, Kareken D, et al. Cognitive deficits in chronic heart failure. Nurs Res. 2010;59(2):127.

5. Qiu C, Winblad B, Marengoni A, Klarin I, Fastbom J, Fratiglioni L. Heart failure and risk of dementia and Alzheimer disease: a populationbased cohort study. Arch Intern Med. 2006;166(9):1003.

6. Hawkins LA, Kilian S, Firek A, Kashner TM, Firek CJ, Silvet H. Cognitive impairment and medication adherence in outpatients with heart failure. Heart Lung. 2012;41(6):572-582.

7. Riegel B, Lee CS, Ratcliffe SJ, et al. Predictors of objectively measured medication nonadherence in adults with heart failure. Circ Heart Fail. 2012;5(4):430-436.

8. Alosco ML, Spitznagel MB, van Dulmen M, et al. Cognitive function and treatment adherence in older adults with heart failure. Psychosom Med. 2012;74(9):965-973.

9. Choudhry NK, Glynn RJ, Avorn J, et al. Untangling the relationship between medication adherence and post-myocardial infarction outcomes: medication adherence and clinical outcomes. Am Heart J. 2014;167(1):51-58.e5.

10. Fitzgerald AA, Powers JD, Ho PM, et al. Impact of medication nonadherence on hospitalizations and mortality in heart failure. $J$ Card Fail. 2011;17(8):664-669.

11. Riegel B, Knafl GJ. Electronically monitored medication adherence predicts hospitalization in heart failure patients. Patient Prefer Adherence. 2014;8:1.

12. Gerber LM, Mann SJ. Inaccuracy of self-reported low sodium diet. Am J Hum Biol. 2012;24(2):189-191.

13. Loria CM, ObarzanekE, Ernst ND. Choose and prepare foods with less salt: dietary advice for all Americans. $J$ Nutr. 2001;131(2):536S-551S.

14. Eaton SB, Eaton SB 3rd. Paleolithic vs modern diets-selected pathophysiological implications. Eur J Nutr. 2000;39(2):67-70.

15. Heart Failure Society of America. Executive summary: HFSA 2010 comprehensive heart failure practice guideline. J Card Fail. 2010;16(6): $475-539$.

16. Gupta D, Georgiopoulou VV, Kalogeropoulos AP, et al. Dietary sodium intake in heart failure. Circulation. 2012;126(4):479-485.

17. Elliott $P$, Stamler J, Nichols R, et al. Intersalt revisited: further analyses of 24 hour sodium excretion and blood pressure within and across populations. BMJ. 1996;312(7041):1249-1253.

18. Safar ME, Benetos A. Factors influencing arterial stiffness in systolic hypertension in the elderly: role of sodium and the renin-angiotensin system. Am J Hypertens. 2003;16(3):249-258.

19. Felder RA, Jose PA. Mechanisms of disease: the role of GRK4 in the etiology of essential hypertension and salt sensitivity. Nat Clin Pract Nephrol. 2006;2(11):637-650.
20. Stone NJ, Merz CN, ScM F, et al. 2013 ACC/AHA guideline on the treatment of blood cholesterol to reduce atherosclerotic cardiovascular risk in adults. $J$ Am Coll Cardiol. 2014;63(25 Pt B): 28889-2934.

21. Yancy CW, Jessup M, Bozkurt B, et al. 2013 ACCF/AHA guideline for the management of heart failure: a report of the American College of Cardiology Foundation/American Heart Association task force on practice guidelines. J Am Coll Cardiol. 2013;62(16): e147-e239.

22. Basuray A, Dolansky M, Josephson R, et al. Dietary sodium adherence is poor in chronic heart failure patients. J Card Fail. 2015;21(4): 323-329.

23. Lennie TA, Worrall-Carter L, Hammash M, et al. Relationship of heart failure patients' knowledge, perceived barriers, and attitudes regarding low-sodium diet recommendations to adherence. Prog Cardiovasc Nurs. 2008;23(1):6-11.

24. Lennie TA, Song EK, Wu JR, et al. Three gram sodium intake is associated with longer event-free survival only in patients with advanced heart failure. J Card Fail. 2011;17(4):325-330.

25. Nasreddine ZS, Phillips NA, Bédirian V, et al. The Montreal Cognitive Assessment, MoCA: a brief screening tool for mild cognitive impairment. J Am Geriatr Soc. 2005;53(4):695-699.

26. Folstein MF, Folstein SE, McHugh PR. "Mini-mental state": a practical method for grading the cognitive state of patients for the clinician. J Psychiatr Res. 1975;12(3):189-198.

27. Hawkins MA, Gunstad J, Dolansky M, et al. Greater body mass index is associated with poorer cognitive functioning in male heart failure patients. J Card Fail. 2014;20:199-206.

28. Teng E, Chui H. The modified mini-mental state examination (3MS). Can J Psychiatry. 1987;41(2):114-121.

29. Blais MA, Baity MR. A comparison of two mental status examinations in an inpatient psychiatric sample. Assessment. 2005;12(4):455-461.

30. Golden JC. Stroop Color and Word Test. Chicago, IL: Stoelting; 1978.

31. Reitan RM. Validity of the trail making test as an indicator of organic brain damage. Percept Mot Skills. 1958;8(3):271-276.

32. Wechsler D. Wechsler Adult Intelligence Scale-Third Edition. San Antonio, TX: The Psychological Corporation; 1997.

33. Dubois B, Slachevsky A, Litvan I, Pillon B. The FAB: a frontal assessment battery at bedside. Neurology. 2000;55(11):1621-1626.

34. Lezak MD. Neuropsychological Assessment. New York, NY: Oxford University Press; 1995.

35. Mertler CA. Interpreting Standardized Test Scores: Strategies for Data-Driven Instructional Decision Making. Thousand Oaks, CA: Sage Publications, Inc.; 2007.

36. James GD, Sealey JE, Alderman M, et al. A longitudinal study of urinary creatinine and creatinine clearance in normal subjects race, sex, and age differences. Am J Hypertens. 1988;1(2):124-131.

37. Gibson RS. Principles of Nutritional Assessment. New York, NY: Oxford University Press; 2005.

38. Espeland MA, Kumanyika S, Wilson AC, et al. Statistical issues in analyzing 24-hour dietary recall and 24-hour urine collection data for sodium and potassium intakes. Am J Epidemiol. 2001;153(10):996-1006.

39. Charlson ME, Pompei P, Ales KL, MacKenzie CR. A new method of classifying prognostic comorbidity in longitudinal studies: development and validation. J Chronic Dis. 1987;40(5):373-383.

40. Dolgin M. Nomenclature and Criteria for Diagnosis of Diseases of the Heart and Great Vessels. New York Heart Association. Boston, MA: Little Brown \& Co; 1994.

41. Roux AV, Merkin SS, Arnett D, et al. Neighbourhood of residence and incidence of coronary heart disease. $N$ Engl J Med. 2001;345(2) :99-106.

42. Rawson K, Gunstad J, Hughes J, et al. The METER: a brief, selfadministered measure of health literacy. J Gen Intern Med. 2010;25(1): $67-71$.

43. Kroenke K, Spitzer RL. The PHQ-9: a new depression diagnostic and severity measure. Psychiatr Ann. 2002;32(9):1-7. 
44. Pilkonis PA, Choi SW, Reise SP, et al. Item banks for measuring emotional distress from the Patient-Reported Outcomes Measurement Information System (PROMIS ${ }^{\circledR}$ ): depression, anxiety, and anger. Assessment. 2011;18(3):263-283.

45. Zimet GD, Powell SS, Farley GK, Werkman S, Berkoff KA. Psychometric characteristics of the multidimensional scale of perceived social support. J Pers Assess. 1990;55(3-4):610-617.

46. Gure TR, Blaum CS, Giordani B, et al. Prevalence of cognitive impairment in older adults with heart failure. J Am Geriatr Soc. 2012; 60(9):1724-1729.

47. Vogels R, Scheltens P, Schroeder-Tanka JM, Weinstein HC. Cognitive impairment in heart failure: a systematic review of the literature. Eur J Heart Fail. 2007;9(5):440-449.

48. O'Brien JT. Vascular cognitive impairment. Am J Geriatr Psychiatry. 2006;14(9):724-733.

49. Cameron J, Worrall-Carter L, Page K, Riegel B, Lo SK, Stewart S. Does cognitive impairment predict poor self-care in patients with heart failure? Eur J Heart Fail. 2010;12(5):508-515.

50. Hajduk AM, Lemon SC, McManus DD, et al. Cognitive impairment and self-care in heart failure. Clin Epidemiol. 2013;5:407.
51. Dickson VV, Lee CS, Riegel B. How do cognitive function and knowledge affect heart failure self-care? J Mixed Methods Res. 2011;5(2): 167-189.

52. Bentley B. A review of methods to measure dietary sodium intake. J Cardiovasc Nurs. 2006;21(1):63-67.

53. van der Wal MH, Jaarsma T, Moser DK, Veeger NJ, van Gilst WH, van Veldhuisen DJ. Compliance in heart failure patients: the importance of knowledge and beliefs. Eur Heart J. 2006;27(4):434-440.

54. Davis KK, Himmelfarb CR, Szanton SL, Hayat MJ, Allen JK. Predictors of heart failure self-care in patients who screened positive for mild cognitive impairment. J Cardiovasc Nurs. 2015;30(2):152-160.

55. Bennett SJ, Huster GA, Baker SL, et al. Characterization of the precipitants of hospitalization for heart failure decompensation. Am J Crit Care. 1998;7(3):168-174.

56. Frediani JK, Reilly CM, Higgins M, Clark PC, Gary RA, Dunbar SB. Quality and adequacy of dietary intake in a southern urban heart failure population. J Cardiovasc Nurs. 2013;28(2):119.
Patient Preference and Adherence

\section{Publish your work in this journal}

Patient Preference and Adherence is an international, peer-reviewed, open access journal that focuses on the growing importance of patient preference and adherence throughout the therapeutic continuum. Patient satisfaction, acceptability, quality of life, compliance, persistence and their role in developing new therapeutic modalities and compounds to optimize

\section{Dovepress}

clinical outcomes for existing disease states are major areas of interest for the journal. This journal has been accepted for indexing on PubMed Central. The manuscript management system is completely online and includes a very quick and fair peer-review system, which is all easy to use. Visit http://www. dovepress.com/testimonials.php to read real quotes from published authors. 